\title{
Why is Homeopathy the Medication of Choice in the Treatment of All Kinds of Diseases Nowadays?
}

\section{Huang Wei Ling*}

Infectious Diseases, General Practice, Nutrition, Acupuncture and Pain

Management. Medical Acupuncture and Pain Management Clinic, Franca, São

Paulo, Brazil

*Corresponding Author: Huang Wei Ling, Infectious Diseases, General Practice, Nutrition, Acupuncture and Pain Management. Medical Acupuncture and Pain Management Clinic, Franca, São Paulo, Brazil.
Received: September 21, 2021

Published: October 18, 2021

(C) All rights are reserved by Huang Wei Ling.
Since the implementation of the Flexner report in 1910, only drugs produced by the pharmaceutical industries were considered scientific [1-3].

And all medications that treated in a more natural way, like floral, homeopathy, herbal medicine were considered non-scientific medications [1-3].

Bleeding practices performed by psychiatrists in North America came to be considered unscientific after this implementation and the physicians needs to prescribe psychotropic medications for most psychiatric illnesses [1-3].

But throughout the history of medicine, we can observe that there were many cultures and many other types of treatments that were forgotten or considered unscientific after the implantation of the Flexner report in 1910 [4].

There was a modernization of the world with the implementation of high technologies in many scientific areas, but everything that is implemented can have consequences for our lives [5].

Since the studies of physics, as carried out by the German physicist Albert Einstein (1879 - 1955) and Capra (1939) in his book Tao da Physics, published in 1975, they say that our entire universe is made of energy, just as the human being is, and therefore everything is made of energy $[6,7]$.
According to traditional Chinese medicine (which has been in existence for over 5,000 years), to the human beings healthy, we need to have Yin, Yang, Qi and Blood energy in balanced state [8].

There is energy in our organs and the production of each energy it is the responsibility of all our five massive internal organs. For example, Liver is responsible for the distribution of $Q i$, Heart is responsible for controlling the Blood flow inside the blood vessels, Spleen is responsible for the absorption of nutrients and production of Blood), Lungs is responsible for the distribution of $Q i$ and Kidney is responsible for the production of Yin, Yang and the deficiency of these energies leads to formation of a variety of emotional and physical disease [8].

The author has been studying the energy pattern of her patients since 2010 because, according to Hippocrates (c. 460 BC-c. 375 $\mathrm{BC}$ ), we should treat our patient, and not the disease the patient is presenting. In several scientific publications written by the author, she usually presents a clinical case in which she attended in 2006 and which represents the model and example of where the author bases all her treatments today and her research in the most diverse areas of the medicine [9].

The person that she attended in 2006 was a 70 -year-old-male patient that was experiencing pain in the lower limbs and had been using anti-inflammatory drugs without any improvement in his condition for 6 months $[8,9]$. 
He sought help to try another type of treatment and the author treated his energy imbalances (showed as energy deficiency in the root of the metaphor of tree) because he had Kidney Yang deficiency and after this treatment, the pain in the lower limbs ceased $[8,9]$.

After 10 sessions of acupuncture, the patient came back to be re-evaluated. He said that his intraocular pressure had improved, which for 40 years had been around $40 \mathrm{mmHg}$ and that it had dropped to $17 \mathrm{mmHg}$ after treatment with acupuncture, for the treatment of lower limb pain. At that time, the patient had not told the doctor that he was being treating for glaucoma for the last 40 years and with no improvement of his condition [8,9].

Thus, the author presented this study entitled Acupuncture Viewed Holistically Can Treat All the Patient's Symptoms at The Same Time, at the Acupuncture Research Congress in 2015, demonstrating whether when we treat energy imbalances, at the tree root level, all symptoms and diseases improved at the same time, even if the physician does not know that the patient has such symptom $[8,9]$.

In another article written by Huang (2021) entitled Is the Population in the World the Same as in the Past?, the author demonstrates that patients she has been treating since 2015 have been showing changes in the energy pattern in all chakras' energy centers, and this results were published in the article written by Huang (2021) entitled Energy Alterations and Chakras' Energy Deficiencies and Propensity to SARS-CoV-2 Infection [10].

This topic can be a little difficult for the Western medicine doctor because in all her articles, the author is talking about the energy alterations found in all her patients, that current medicine does not study yet, as this type of medicine is studied by older medicine's such as traditional Chinese medicine [4].

In this article, the author will try to integrate the two medicines (Western and Chinese) that had to be separated after the implementation of the Flexner report in 1910 (where Western medicine studies the materialized form of disease formation and Chinese medicine studies the energy alterations that is leading to the formation of variety of diseases before materializing) and today's physician had to reason in front of his patient just observing the materialized form, after the implementation of Flexner report, as the other medicines that dealt with energy point of view were considered unscientific. For this reason, the first energy alterations that is the first three phases of energy imbalances from health to disease formation is not discussed and the physician nowadays has difficulties in doing this earlier diagnosis and only doing diagnosis in the later phase of disease formation in the phase four or five of energy alterations) where the energy imbalances are leading to alterations in the laboratory and or radiological level, after 5 or 10 years after the energy imbalances. All these explanations were done by Huang (2019) in the article entitled Why Are Diabetic Patients Still Having Hyperglycemia Despite Diet Regulation, Antiglycemic Medication and Insulin? $[8,11]$.

Flexner was not a doctor but only a professor and after his reforms in all medical faculties in the world, now after more than 100 years of its implementation, positive points had with these reforms but also negative points are appearing with the passage of time, as our world has changed, with innovations and technologies, especially in the area of telecommunications, with the use of cell phones, computers, producing negative influences. These influences in the human body could be explained by the chronic exposition to electromagnetic radiation, as the author demonstrates in the article written by Huang (2021) entitled Energy Alterations and Chakras' Energy Deficiencies and Propensity to SARS-CoV-2 Infection. In this article, the author analyzed the chakras' energy alterations of her patients over a period of 2015 to 2020, evaluating a thousand patient's chakras' energy centers, and it was observed that $90 \%$ of patients have no energy in the five internal massive organs that are represented by Liver, Heart, Spleen, Lung and Kidney [11,12].

The results of the remaining $10 \%$ of the patients analyzed were published in an article written by Huang (2021) entitled Are We Vaccinating Immunocompetent or Immunocompromised People for COVID 19?, where the author demonstrates that $97 \%$ of patients have Spleen energy deficiency (fifth chakra), which is responsible for nutrient absorption and Blood production (red and white cells) and $97 \%$ of patients have Kidney energy deficiency (second chakra), which is responsible, among other functions, for the production of Zheng-Qi, which is the energy that protects the individual against the invasion of external pathogenic factor, in this case, against the invasion of SARS-CoV-2 into the human body $[13,14]$.

In the article written by Huang (2021) entitled Are We Vaccinating Immunocompetent or Immunocompromised People for CO- 
VID-19? the author did a research from 2015 and 2020, studying a 1000 patient's chakras' energy centers and what she found in her study was that more than $90 \%$ of her patients do not have any energy in the five internal massive organs. And in this study, she demonstrated that the organs that are responsible for the distribution of energy inside the body are Liver (first chakra) and Lung (fourth chakra) and in this study, she is demonstrating that the 98,32\% of the population she is attending do not have energy in the Liver (first chakra). And also, 98,08\% of the population do not have any energy in the Lung (fourth chakra). In this same study, the author is showing that $97 \%$ of the patients do not have any energy in the Kidney (second chakra) that is responsible for the production of Yin and Yang energy. Also, she demonstrated that $97 \%$ of the patients do not have energy in the Spleen, responsible for the production of Blood, and for this reason, all the energies that are important to maintain health to the patients are all very compromised in this population $[12,13]$.

According to this result, you can notice that the population that the author is attending quite do not have energy to do the distribution of energy in the entire body, predisposing the patient to have Blood clot and stagnation of energy inside the meridians. This is very important to understand because many symptoms that are occurring in the patients nowadays cannot be explained by the laboratory exams because all the alterations are in the energy level (that we cannot see by the naked eyes). For example, many cases of thrombosis after COVID-19 vaccination cannot be explained by the laboratory exams. The vaccine is considered a highly concentrated medication and according to Arndt Shultz Law, created in 1888, by two German researchers, they said that the use of highly concentrated medications will decrease the vital energy of the patient and if we reduce even more the energy of our patients that are very low, it will cause many side effects such as stagnation of this energy and Blood inside de energy meridians and inside the blood vessels, leading to Blood stagnation and symptoms of myocardial infarction or stroke or stagnation of Blood in any site of the body $[13,15,16]$.

But if we follow Hippocrates (460 bce-375 bce), father of medicine, he said that we need to treat the patient and not the diseases [17].

Under these conditions in which the energy of her patients in Brazil is very low, the author makes a correlation of these results with a probable state of the world population in this same state, as this reduction in energy is related to the exposition to electromagnetic waves, that quite all entire population in this planet is suffering nowadays $[5,12]$.

Since 2018, the author has been writing articles in a variety of specialties where she writes about emotional, psychiatric and physical illnesses in babies, children, adolescents, young adults and elderly patients, and in all her articles, what these patients have in common today, is the energy deficiency of the chakras' energy centers [8,18-21].

For this reason, to increase the formation of this vital energy, which is compromised in all these patients, regardless of race, age, presence of comorbidity or not, we must use highly diluted medications, as showed in the Arndt-Shultz law. According to them, highly concentrated medications reduce vital energy, which can cause many side effects and cause further reduction of this energy that is very low nowadays, and which can lead to the formation of other diseases, or even lead to death, as demonstrated by this law and published on may article written by Huang [16].

Thus, the author would like to write in this article, that we need to go through more than 100 years of implementation of the Flexner report, to know that forced measures in the past are causing serious consequences in relation to the way and type of medicine used in these days today, and which are taught by medical schools around the world [11].

Therefore, reassessing and expanding our field of vision is very important for us to understand that the human being is part of this universe, and that before becoming matter, it is formed by a constant transformation of Yin and Yang energy and the understanding of the formation of diseases, since the energy imbalances, it is of paramount importance to know which kind of medication we should treat the patient nowadays. According to one theme presented by the author, in the $9^{\mathrm{TH}}$ Webinar On Healthcare- Health Economics and Policy, that was held on February 09, 2021, in London (UK), entitled To Know Which Medicine We Should Prescribe, We Need To Know What Type of Patient We Have Nowadays [7,22].

To treat this condition of lack of energy in the internal massive organs, the author is using homeopathy medications but according to the theory created by Huang (2021) entitled Constitution- 
al Homeopathy of the Five Elements Based on Traditional Chinese Medicine. This theory was created by the author when she began to study homeopathy in Brazil in 2015. During this course, besides she noticed that homeopathy created by Hahnemann (1755-1843) 242 years ago, this kind of medication is a very effective treatment for the curing of variety of diseases nowadays, and the physician that are using this mode of treatment, still do not understand the mechanism of action of this kind of approach. According to Hahnemann, if you give a substance for a healthy individual and he becomes sick due to ingestion of this substance, if you dilute this substance in a very diluted form, this substance can treat this condition, in a simillimum theory [23].

In China, this condition of lack of energy inside the internal organs is treated using Chinese herbs but the author has used Chinese herbal medicines only in an infinitesimally diluted way, in order to increase the vital energy of her patients, as if taken in a highly concentrated way, such as recommended for years, the patient can have serious side effects nowadays, such as the formation of internal Heat and generating other diseases, due to the different energy pattern of our population nowadays, as demonstrated in the article Is the population the same in the past? [10].

As Hippocrates said, we must treat the patient and not the disease. It is very important nowadays to treat the energy deficiencies of the five massive organs, in all patients globally, treating diseases at the root of their formation, through the theory Constitutional Homeopathy of the Five Elements based on Traditional Chinese Medicine, in which the author makes a link between homeopathy and Chinese medicine, because the author showed in all her articles published to treat the majority of diseases nowadays, are related to the lack of energy in the five internal massive organs and the replenishment of this energy, will treat all conditions at the same time, even the physician does not know that the patient have such symptoms $[9,17,23]$.

According to Albert Einstein (1879-1955), we cannot solve our problems with the same thinking we used when we created them [6].

\section{Bibliography}

1. Stahnisch FW and Verhoef M. "The Flexner Report of 1910 and Its Impact on Complementary and Alternative Medicine and
Psychiatry in North America in the 20th Century". EvidenceBased Complementary and Alternative Medicine 2012 (2012): $1-10$.

2. Duffy TP., et al. "The Flexner Report - 100 Years Later". Yale Journal of Biology and Medicine 84 (2011): 269-276.

3. Aurélio M and Ros D. "The Flexner Repor: for Good and for Bad”. Revista Brasileira de Educação Médica 32.4 (2008): 492499.

4. Huang Wei Ling. "What Do All Autoimmune Diseases Have in Common?". Clinical and Experimental Immunology 6.3 (2021): 301-304.

5. Huang WL. "The Influence of Cell Phones and Computers on Our Immune System". Annals of Immunology and Immunotherapy 3.2 (2021): 000141.

6. Albert Einstein. Mac Tutor (2021).

7. Capra F. "The Tao of Physics". 5th ed. Shambhala Publications (2010): 368.

8. Huang Wei Ling. "Why Are Diabetic Patients Still Having Hyperglycemia Despite Diet Regulation, Antiglycemic Medication and Insulin?" International Journal of Diabetes and Metabolic Disorders 4.2 (2019): 1-14.

9. Huang Wei Ling. "The Importance of Correcting Energy Imbalances and Chakras Energy Deficiencies in the Treatment of Patients with Glaucoma". Clinical Research on Ophthalmology 2.2 (2019): 1-9.

10. Huang Wei Ling. "Is the Population in the World the Same as in the Past?". Acta Scientific Clinical Case Reports 2.6 (2021).

11. Huang Wei Ling. "What Flexner Report did to Our Medicine After 100 Years of Implantation?". Acta Scientific Gastrointestinal Disorders 4.8 (2021): 01-04.

12. Huang Wei Ling. "Energy Alterations and Chakras' Energy Deficiencies and Propensity to SARS-CoV-2 Infection". Acta Scientific Microbiology 4.4 (2021): 167-196.

13. Huang WL. "Are We Vaccinating Immunocompetent or Immunocompromised People for COVID 19?" Journal of Vaccines Research and Vaccination 7 (2021): 018.

Citation: Huang Wei Ling. "Why is Homeopathy the Medication of Choice in the Treatment of All Kinds of Diseases Nowadays?". Acta Scientific Medical Sciences 5.11 (2021): 66-70. 
14. Huang WL. "Is SARS-CoV- 2 Strong or Our Body Is Weak?" Journal of Virology 1.1 (2021).

15. Huang Wei Ling. "What We Need to Know When the Patient has a Stroke with or without COVID-19?". Acta Scientific Neurology 4.8 (2021): 01-05.

16. Mastrangelo D. "Hormesis, epitaxy, the structure of liquid water, and the science of homeopathy". Medical Science Monitor 13.1 (2007): 1-8.

17. Craik E. The "Hippocratic" Corpus: Content and Context. Routledge (2014): 344.

18. Huang Wei Ling. “Chakras' Energies Deficiencies as the Main Cause of Myocardial Infarction without Arterial Obstruction". Online Journal of Cardiology Research and Reports 4.5 (2021).

19. Huang Wei Ling. "The Importance of Correcting Energy Imbalances in the Prevention and Treatment of Myocardial Infarction". Acta Scientific Medical Sciences 4.6 (2020): 20-27.

20. Huang Wei Ling. "Energy Alterations as the Underlying Cause of Primary Hypertension". ARC Journal of Nephrology 4.2 (2019): 33-44.

21. Huang Wei Ling. "Energy Disturbances in Panic Syndrome and How Can We Teat It in Adolescence?" Journal of Neurology and Experimental Neuroscience 3.1 (2020): 139.

22. 9TH Webinar On Healthcare- Health Economics and Policy. February 09, 2021 London UK (2021).

23. Huang Wei Ling. "Constitutional Homeopathy of Five Elements Based on Traditional Chinese Medicine". Acta Scientific Medical Sciences 4.7 (2020): 57-69.

Volume 5 Issue 11 November 2021

C) All rights are reserved by Huang Wei Ling. 